\title{
Size-Dependent Surface Energy Density of Spherical Face-Centered-Cubic Metallic Nanoparticles
}

\author{
Yaochi Wei and Shaohua Chen* \\ LNM, Institute of Mechanics, Chinese Academy of Sciences, Beijing 100190, China
}

\begin{abstract}
The surface energy density of nano-sized elements exhibits a significantly size-dependent behavior. Spherical nanoparticle, as an important element in nano-devices and nano-composites, has attracted many interesting studies on size effect, most of which are molecular dynamics (MD) simulations. However, the existing MD calculations yield two opposite size-dependent trends of surface energy density of nanoparticles. In order to clarify such a real underlying problem, atomistic calculations are carried out in the present paper for various spherical face-centered-cubic (fcc) metallic nanoparticles. Both the embedded atom method (EAM) potential and the modified embedded atom method (MEAM) one are adopted. It is found that the size-dependent trend of surface energy density of nanoparticles is not governed by the chosen potential function or variation trend of surface energy, but by the defined radius of spherical nanoparticles in MD models. The finding in the present paper should be helpful for further theoretical studies on surface/interface effect of nanoparticles and nanoparticle-reinforced composites.
\end{abstract}

Keywords: Nanoparticle, Surface Energy Density, Size Effect, Molecular Dynamics Simulation.

\section{INTRODUCTION}

Surface effect in nanomaterials such as nanofilm, nanowire and nanoparticle has attracted considerable attention for years. One of the major outcomes of surface effect is that physical and chemical properties as well as mechanical behaviors of nanomaterials distinguish significantly from their macroscopic counterparts. This distinction mainly results from the relatively large surface-to-volume ratio of nanomaterials comparing to the bulk ones. ${ }^{1}$ The surface atoms with fewer neighbors and consequently excess energies over atoms in the bulk can greatly affect the overall performance of nanostructures, for example, melting behaviors, ${ }^{2}$ thermal conductivity, ${ }^{3}$ and the elastic behaviors, ${ }^{4}$ etc.

The most importantly physical quantity characterizing surface effect is the surface free energy density, which is a reversible work necessary to create a unit of new surface area. ${ }^{5}$ In the existing continuum mechanics models considering surface effect in nanomaterials, ${ }^{6-8}$ surface free energy density is related to the deformation of nanomaterial surface and its derivative with respect to the surface strain leads to the surface stress. One of the representative works is the Gurtin and Murdoch model

*Author to whom correspondence should be addressed.
(G-M theory). ${ }^{9}{ }^{10}$ Analytical expressions of surface energy density with Lagrangian and Eulerian descriptions were derived by Nix and Gao. ${ }^{11}$

However, experimental measurement of surface free energy density of nanomaterials is still trammeled even to this day due to the low characteristic scale. Instead, $a b$ initio and molecular dynamics (MD) simulations are adopted as effective approaches, with which surface energy densities of several face-centered-cubic (fcc) and body-centered-cubic (bcc) metals were achieved. ${ }^{12-17}$ Size dependent features of various fcc metallic nano-slabs have been discussed by Zhang et al. ${ }^{18}$ in which not only the Lagrangian and Eulerian surface energy densities but also the surface relaxation parameters were calculated as a function of the nanoslab thickness.

In contrast to the well-studied nanostructures with plane surface (for example, nanofilms and nano-slabs), nanoparticles, as an important element in design of nano-devices and nanocomposites, possess hybrid crystal faces, which lead to more challenges in determining surface properties. Theoretical approach to surface energy of nanoparticles with continuum hypothesis is merely approximate in providing surface structures and its properties. ${ }^{19} \mathrm{MD}$ simulation is always adopted in practice to find the size effect of surface energy density of nanoparticles..$^{20-23}$ However, 


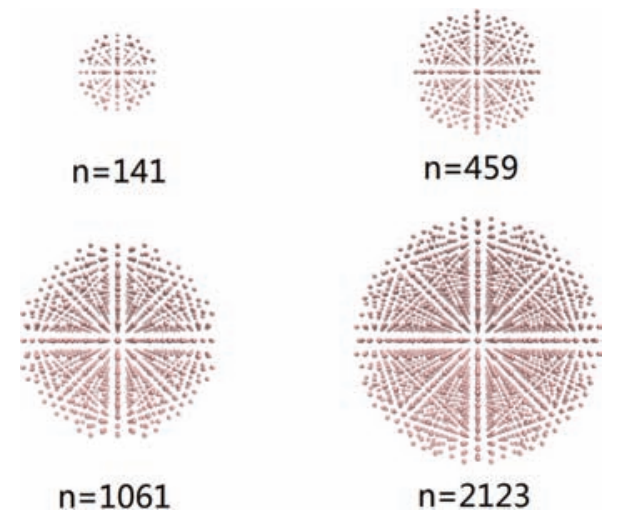

Figure 1. Atomic models of spherical nanoparticles cut from a bulk material with different radii for MD simulation, where $n$ is the atomic number in each nanoparticle.

the existing MD calculations for nanoparticles yield two opposite trends of surface energy density in size-dependent behavior. For example, size effects of $\mathrm{Ag}$ and $\mathrm{Al}$ spherical nanoparticles were studied by Medasani et al. ${ }^{20,21}$ in which density functional theory (DFT) calculations were carried out for nanoparticles with radius below $10 \mathrm{~nm}$ and MD simulations with EAM potential were conducted for nanoparticles with radius above $10 \mathrm{~nm}$. A downward trend of surface energy density as a function of the increasing nanoparticle size was observed. ${ }^{20,21}$ The same conclusion for $\mathrm{Cu}$ nanoparticles resulting from $\mathrm{MD}$ simulation with EAM potential was made by Bian et al. ${ }^{22}$ However, Luo et al. ${ }^{23}$ employed a modified analytic embedded atom method (MAEAM) in MD simulation and found an upward trend of surface energy density varying with the increasing size of $\mathrm{Ni}$ nanoparticles. Apparently, the potential function may be a governing role on the varying trend. Is it true? If not, what factor leads to such different trends?

MD simulations with both $\mathrm{EAM}^{24,25}$ and modified embedded atom method (MEAM) $)^{26,27}$ are carried out in the present paper for nickel, platinum, palladium, copper, gold, and silver nanoparticles. Not only the surface energy

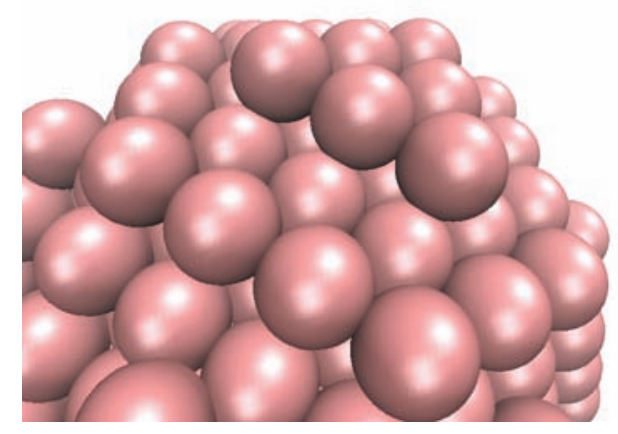

Figure 2. Schematic of a Ag nanoparticle after relaxation in MD simulation, where the surface is not perfectly spherical. but also the surface energy density is calculated for various fcc metallic nanoparticles. In order to achieve surface energy density from the calculated surface energy, different definitions of radius are adopted for spherical nanoparticles. Factors leading to different size-dependent trends of surface energy density of nanoparticles are discussed. The finding in the present paper should be helpful for clarifying size effect of nanoparticles and the forthcoming theoretical studies on mechanical analysis of nanoparticles and nanocomposites.

\section{MD SIMULATION MODEL AND DEFINITION OF RADIUS}

\subsection{The Atomic Model}

The atomic model of a nanoparticle in MD simulation is shown in Figure 1, which is generated by Boolean operation, i.e., removing atoms outside a determined spherical face with specified radius from an intact cubic crystalline metal material. This leads to smooth but faceted surface structures shown in Figure 2.

Both EAM potential $^{24,25}$ and MEAM potential ${ }^{26,27}$ are adopted to describe interactions among atoms in our simulations. The results of MAEAM potential given by Luo et al. $^{23}$ will be compared and discussed at the end of this paper.

In the embedded atom formalism (EAM), the total energy $E$ can be written as, ${ }^{24,25}$ ry

$$
E=\sum_{i} F_{i}\left(\rho_{i}\right)+\frac{1}{2} \sum_{i \neq j} \phi_{i j}\left(r_{i j}\right) \quad \rho_{i}=\sum_{i \neq j} \rho_{a}\left(r_{i j}\right)
$$

where $F_{i}$ is the embedding energy needed to place atom $i$ in a local electron density $\rho_{i}$ and $\phi_{i j}$ is the pair interaction between atoms $i$ and $j$ with a distance $r_{i j} . \rho_{a}$ is the spherically averaged atomic electron density of an atom at a

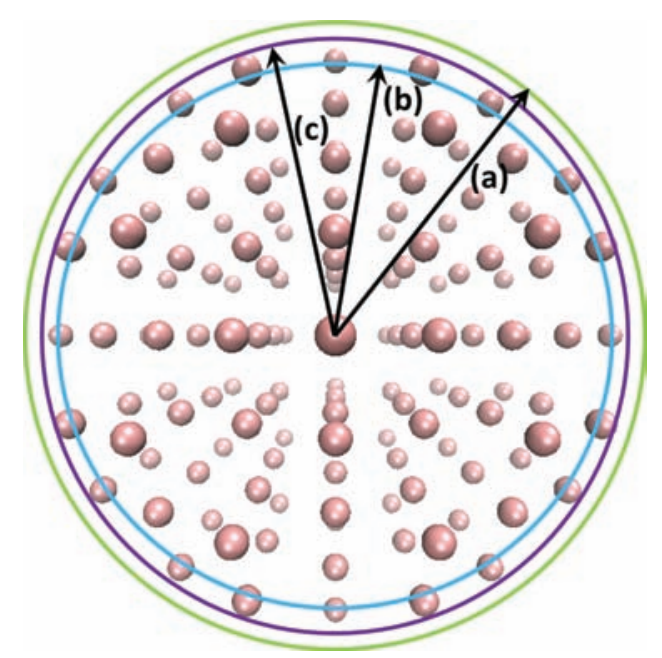

Figure 3. Three kinds of definitions of radius of nanoparticles, which are denoted by (a), (b) and (c).

J. Nanosci. Nanotechnol. 15, 9457-9463, 2015 

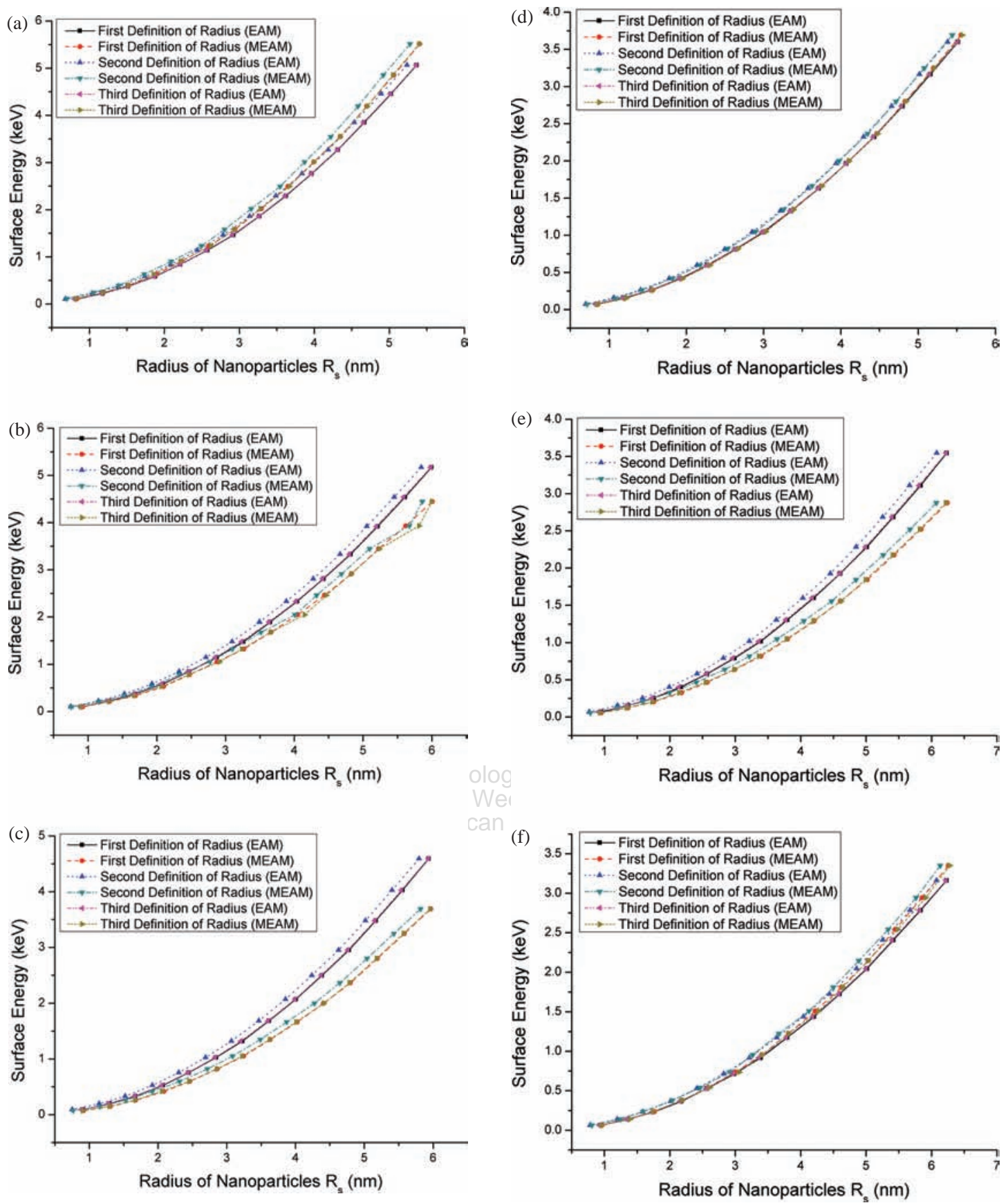

Figure 4. Surface energy of various metallic fcc nanoparticles calculated with EAM and MEAM potentials as a function of different defined radii. (a) for $\mathrm{Ni}$; (b) for Pt; (c) for Pd; (d) for $\mathrm{Cu}$; (e) for $\mathrm{Au}$; (f) for $\mathrm{Ag}$.

distance $r_{i j}$ from the nucleus. ${ }^{28}$ It should be noted that the total energy $E$ is usually regarded as an additional energy with respect to the un-deformed lattice with a zero energy assumption per atom. ${ }^{15}$

The total energy in MEAM ${ }^{26,27}$ has the same formula as Eq. (1), but with a different definition of the local electron density $\rho_{i}$ as follows,

$$
\rho_{i}=\sum_{i \neq j} \rho_{a}\left(r_{i j}\right)-a \sum_{\substack{j \neq i \\ k \neq i}}\left(1-3 \cos ^{2} \theta_{i j k}\right) \rho_{a}\left(r_{i j}\right) \rho_{a}\left(r_{i k}\right)
$$

where an additional term, in contrast to EAM, is added in order to improve the accuracy of EAM. $\theta_{i j k}$ is an included angle among atoms $i, j$, and $k ; a$ is a constant to be determined by fitting to the shear moduli. On the right side of Eq. (2), the first term is a linear superposition of atomic densities and the second one is related to angular correction. $^{29}$

LAMMPS $^{30}$ is used in all MD simulations. Nanoparticles cut from a bulk crystal are first relaxed by iteratively adjusting atom coordinates to achieve a minimum local 
potential energy. Afterwards, sufficient relaxation is conducted at a constant temperature $(10 \mathrm{~K})$ and a constant atmospheric pressure (1.0 bar) with Nose-Hoover thermostat and barostat. When the equilibrium state of the simulation system is achieved, the data, such as the surface energy and the radius of nanoparticles, will be recorded.

\subsection{Surface Energy Density and Spherical Radius}

According to Ref. [18,31] where a thin nanoslab was investigated, the surface energy density for a relaxed nanosystem cut from a bulk can be written as

$$
\gamma=\frac{U_{n s}-n U_{\text {bulk }}}{A}
$$

where $U_{n s}$ is the total potential energy of a nano-system after relaxation. $U_{\text {bulk }}$ is the free energy per atom in a bulk and $n$ is the total atom number in the nano-system. $U_{n s}-n U_{\text {bulk }}$ denotes the total surface free energy at nanoscale. $A$ is the surface area of the nano-system after relaxation.

For a spherical nanoparticle, we have,

$$
A=4 \pi R_{s}^{2}
$$

where $R_{s}$ is the radius of spherical nanoparticles.

To achieve the surface energy density of nanoparticles, it is easy to find both $U_{n s}$ and $U_{\text {bulk }}$ from MD calculations, but how to define the spherical radius is still ambiguous due to an imperfectly spherical surface for nanoparticles $^{23,32}$ as shown in Figure 2. Several different definitions of radius will be used and discussed in the present paper as shown in Figure 3.

(a) The first definition of radius is taken from Qi et al. 23,32

$$
R_{s}=R_{g} \sqrt{5 / 3}+R_{N i}
$$

where $R_{N i}$ is half the atomic distance in bulk materials and $R_{g}$ is the radius of gyration,

$$
R_{g}=\sqrt{\left(\frac{1}{n}\right) \sum_{i}\left|\boldsymbol{R}_{i}-\boldsymbol{R}_{\mathrm{cm}}\right|^{2}}
$$

in which $\boldsymbol{R}_{i}$ denotes the coordinate vector of the $i$ th atom in nanoparticles and $\boldsymbol{R}_{\mathrm{cm}}$ is the vector of mass center of nanoparticles,

$$
\boldsymbol{R}_{\mathrm{cm}}=\frac{1}{M} \sum_{i=1}^{n} m_{i} \boldsymbol{R}_{i}
$$

Here, $m_{i}$ represents the mass of atom $i$ and $M$ is the mass of the nanoparticle. Since the calculated nanoparticle consists of the same atoms, the vector of mass center becomes

$$
\boldsymbol{R}_{\mathrm{cm}}=\frac{1}{n} \sum_{i=1}^{n} \boldsymbol{R}_{i}
$$

(b) The second defined radius is half the distance of two farthest atoms in nanoparticles, the connected line between which should go through the mass center.

(c) Radius of the third definition is equal to the one defined in (b) plus the radius of an atom, as shown in Figure 3.

\section{RESULTS AND DISCUSSION}

Surface energy and surface energy density of different fcc metallic nanoparticles with different sizes, including $\mathrm{Ni}$, $\mathrm{Pt}, \mathrm{Pd}, \mathrm{Cu}, \mathrm{Au}$ and $\mathrm{Ag}$, are calculated with both EAM and MEAM potentials. Here, the system temperature keeps $10 \mathrm{~K}$. Since the surface energy density is relevant to surface energy and radius of nanoparticles, we first analyze size effect of the surface energy of different nanoparticles. Then, the surface energy density will be analyzed using different defined radii to check whether the varying trend of size-dependent surface energy density is consistent with that of the surface energy and whether the size dependent feature of surface energy density depends mainly on the chosen potential function or the definition of radius of nanoparticles.

\subsection{Size Effect of Surface Energy}

The surface energy of different metallic nanoparticles with different sizes is calculated according to $U_{n s}-n U_{\text {bulk }}$. The relation between surface energy and radius of nanoparticles is given in Figures 4(a)-(f) for Ni, Pt, Pd, Cu, $\mathrm{Au}$ and $\mathrm{Ag}$, respectively, where EAM and MEAM potentials are
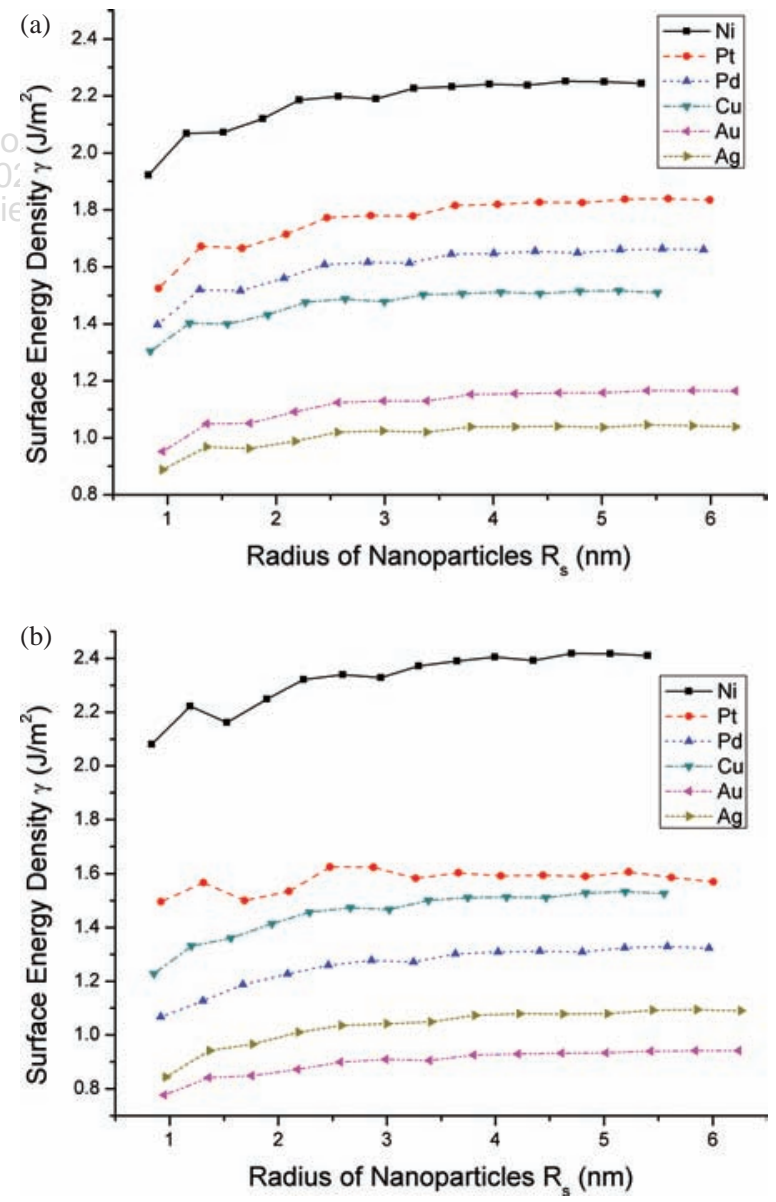

Figure 5. Surface energy density calculated by the first defined radius with two kinds of potential functions, respectively. (a) with EAM potential; (b) with MEAM potential.

J. Nanosci. Nanotechnol. 15, 9457-9463, 2015 

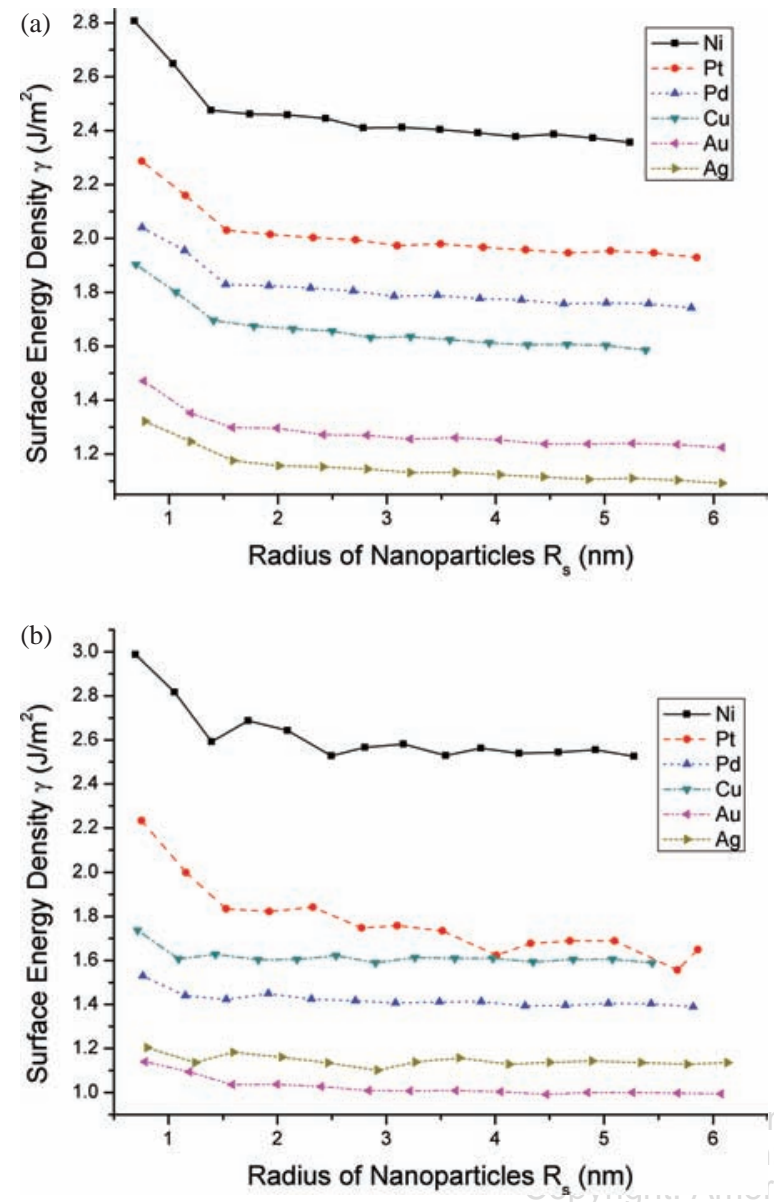

Figure 6. Surface energy density calculated by the second defined radius with two kinds of potential functions, respectively. (a) with EAM potential; (b) with MEAM potential.

used. Radius of nanoparticles is calculated with the above three different definitions. It is found that surface energy of each kind of nanoparticle increases with the increasing size of nanoparticles and the size dependent feature of surface energy for the various metallic nanoparticles keeps consistent, without dependence on the potential functions and definition of radius, though the magnitude of surface energy is influenced slightly by the chosen potential functions.

\subsection{Size Effect of Surface Energy Density}

The surface energy density of different fcc metallic nanoparticles with different radii is calculated according to Eqs. (3) and (4), using different potential functions and different defined radii. Figure 5 gives the surface energy density as a function of the radius of nanoparticles with EAM potential used in Figure 5(a) and MEAM potential used in Figure 5(b), where radius of the first definition is used. The size-dependent trends in Figures 5(a) and (b) are the same, where the surface energy density increases with the increase of radius of nanoparticles. It demonstrates that potential function does not change the size-dependent feature of surface energy density of nanoparticles.

Figure 6 gives the relation between surface energy density and radius of different nanoparticles with EAM potential used in Figure 6(a) and MEAM potential adopted in Figure 6(b). Radius of the second definition is used in both Figures 6(a) and (b). It is interesting to find that the size dependent trend in Figure 6 is totally opposite to that in Figure 5. The surface energy density decreases with the increase of radius of nanoparticles. One should be noted that, to achieve the surface energy density, the unique difference between Figure 5 and Figure 6 is the defined radius.

Figure 7 shows another set of relation between surface energy density and radius of different nanoparticles, in which radius of the third definition is chosen. EAM potential and MEAM one are used in Figures 7(a) and (b), respectively. It is surprising to find that the size-dependent feature of surface energy density of nanoparticles keeps the same as that in Figure 5, but is contrary to that in Figure 6.
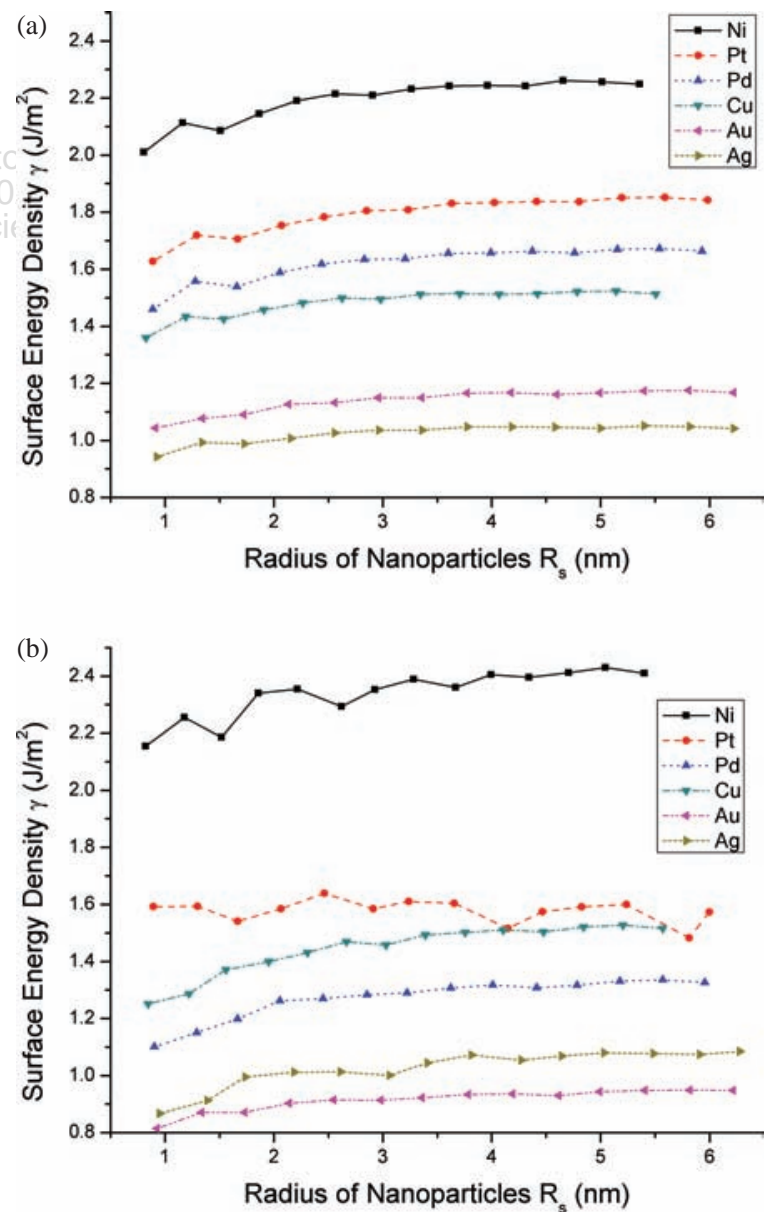

Figure 7. Surface energy density calculated by the third defined radius with two kinds of potential functions, respectively. (a) with EAM potential; (b) with MEAM potential. 


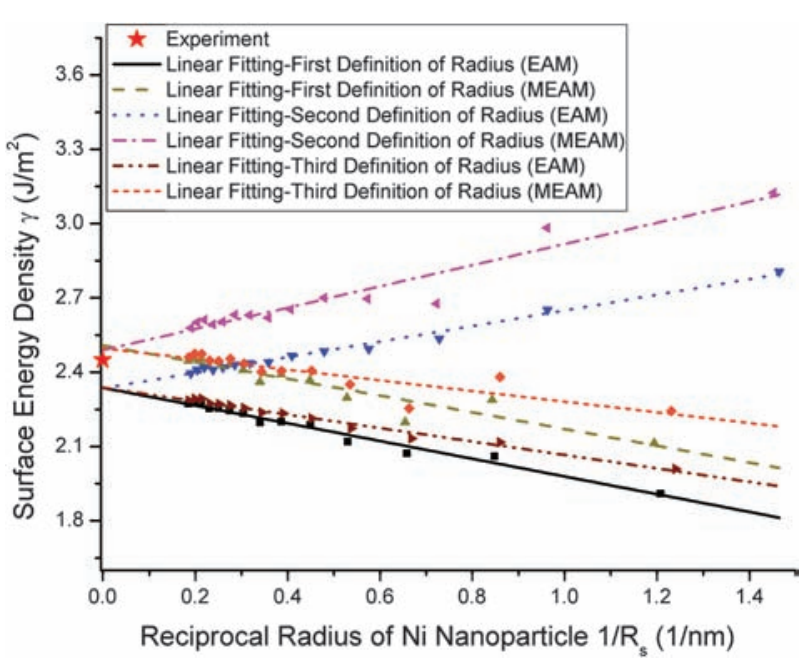

Figure 8. Surface energy density as a function of the reciprocal of radius of Ni nanoparticles, in which the results calculated by two kinds of potentials and three kinds of defined radius are given for comparison with the experimental one ${ }^{33}$ and Luo et al..$^{23}$

\subsection{Discussion}

In the present paper, EAM and MEAM potentials are used in contrast to MAEAM potential suggested by Luo et al. ${ }^{23}$ Comparing the present results to that in Luo et al. ${ }^{23}$ we find that the size-dependent trend of surface energy density is the same only if the defined radius is consistent. This finding denotes that the result is hardly relevant to the chosen potentials. In order to compare with the results given by Luo et al. ${ }^{23}$ in which the calculation was conducted at $0 \mathrm{~K}$ for Ni nanoparticles, we also carry out simulation on $\mathrm{Ni}$ at $0 \mathrm{~K}$ with both EAM and MEAM potentials. Figure 8 gives the surface energy density for Ni nanoparticles as a function of the reciprocal diameter of Ni nanoparticles, in which the above mentioned three kinds of definitions of radius are used and the value of surface energy density for bulk Ni material is shown for comparison. Figure 8 shows a very similar result to that given by Luo et al. ${ }^{23}$ if the first defined radius, which is the same as that taken in Luo et al., or the third defined radius, is used, no matter what potential (EAM, MEAM or MAEAM) is adopted. The size-dependent trend with the second defined radius used is opposite to the other two for both EAM and MEAM potentials, but consistent with that in Ref. [22]. Interestingly, all MD simulation results are consistent well with the experimental one for bulk $\mathrm{Ni}$ when the diameter of nanoparticle is large enough. It further demonstrates that size-dependent trend of surface energy density of nanoparticles does not depend on the chosen potential, but significantly depends on the defined radius for nanoparticles.

\section{CONCLUSIONS}

The main objective of this paper is to clarify the existing opposite size-dependent trends of surface energy density of fcc metallic nanoparticles, in which surface energy density of nanoparticles was found to either increase or decrease with the increase of nanoparticle size. Possible analysis on such an issue is not found so far. MD simulation is carried out with two kinds of potentials, EAM and MEAM, for various fcc metals. Three kinds of defined radii are adopted to analyze MD simulation results, respectively, i.e., radius originated from the one of gyration, half the distance of farthest atoms, and sum of half the distance of farthest atoms and atomic radius. Comparisons with the existing literatures and the experimental results are conducted. All the results show that two opposite size-dependent trends of surface energy density for nanoparticles are not due to the chosen potential, but depend on the definition of radius of nanoparticles. The surface energy density increases with the increase of nanoparticle size if the first or third defined radius is chosen, no matter potential function of EAM, MEAM or MAEAM is used. The varying trend is opposite to the former if the second defined radius in the present paper is adopted. How to define radius of nanoparticles correctly is an obvious question that should be further studied. The most reasonable way to give a reasonable definition of radius of nanoparticles is to compare numerical calculations with expected experiment results for nanoparticles, which may be possible in the future.

Acknowledgments: The work reported here is supported by NSFC through Grants \#11372317, \#11125211, the Nano-project (2012CB937500), and the CAS/SAFEA International Partnership Program for Creative Research Teams.

\section{References and Notes}

1. P. Sharma, S. Ganti, and N. Bhate, Appl. Phys. Lett. 82, 535 (2003).

2. L. Miao, V. R. Bhethanabotla, and B. Joseph, Phys. Rev. B 72, 134109 (2005).

3. L. H. Liang and B. Li, Phys. Rev. B 73, 153303 (2006).

4. R. Dingreville and J. Qu, J. Mech. Phys. Solids 53, 1827 (2005).

5. J. W. Gibbs, The Collected Works of J. Willard Gibbs, Green and Co., New York (1928).

6. R. Shuttleworth, Proc. Phys. Soc. Sect. A 63, 444 (1950).

7. J. S. Vermaak, C. W. Mays, and D. Kuhlmann-Wilsdorf, Surf. Sci. 12, 128 (1968).

8. G. Ouyang, C. X. Wang, and G. W. Yang, Chem. Rev. 109, 4221 (2009).

9. M. Gurtin and A. Ian Murdoch, Arch. Ration. Mech. Anal. 57, 291 (1975).

10. M. E. Gurtin and A. Ian Murdoch, Int. J. Solids Struct. 14, 431 (1978).

11. W. D. Nix and H. Gao, Scr. Mater. 39, 1653 (1998).

12. L. Vitos, A. V. Ruban, H. L. Skriver, and J. Kollar, Surf. Sci. 411, 186 (1998).

13. R. E. Miller and V. B. Shenoy, Nanotechnology 11, 139 (2000).

14. Q. Jiang, D. S. Zhao, and M. Zhao, Acta Mater. 49, 3143 (2001).

15. V. B. Shenoy, Phys. Rev. B 71, 094104 (2005).

16. C. Mi, S. Jun, D. A. Kouris, and S. Y. Kim, Phys. Rev. B 77, 075425 (2008).

17. H. W. Sheng, M. J. Kramer, A. Cadien, T. Fujita, and M. W. Chen, Phys. Rev. B 83, 134118 (2011).

18. C. Zhang, Y. Yao, and S. Chen, Comput. Mater. Sci. 82, 372 (2014).

19. W. G. Wolfer, Acta Mater. 59, 7736 (2011). 
20. B. Medasani, Y. H. Park, and I. Vasiliev, Phys. Rev. B 75, 235436 (2007).

21. B. Medasani and I. Vasiliev, Surf. Sci. 603, 2042 (2009).

22. J. Bian, G. Wang, and X. Feng, Acta Mech. Solida Sin. 25, 557 (2012).

23. W. Luo, W. Hu, K. Su, and F. Liu, Appl. Surf. Sci. 265, 375 (2013).

24. M. S. Daw and M. I. Baskes, Phys. Rev. Lett. 50, 1285 (1983).

25. M. S. Daw and M. I. Baskes, Phys. Rev. B 29, 6443 (1984).

26. M. I. Baskes, Phys. Rev. B 46, 2727 (1992).

27. P. M. Gullett, G. Wagner, and A. Slepoy, Numerical Tools for Atomistic Simulations, Sandia National Laboratories, Albuquerque and Livermore (2004).
28. M. I. Baskes, J. S. Nelson, and A. F. Wright, Phys. Rev. B 40, 6085 (1989).

29. M. I. Baskes, Phys. Rev. Lett. 59, 2666 (1987).

30. LAMMPS, http://lammps.sandia.gov/ (2013).

31. U. Hansen, P. Vogl, and V. Fiorentini, Phys. Rev. B 60, 5055 (1999).

32. Y. Qi, T. Cagin, W. L. Johnson, and W. A. Goddard, J. Chem. Phys. 115, 385 (2001)

33. F. R. de Boer, R. Boom, W. C. M. Mattens, A. R. Miedema, and A. K. Niessen, Cohesion in Metals: Transition Metal Alloys, NorthHolland, Amsterdam (1988).

Received: 20 August 2014. Accepted: 2 September 2014. 